\title{
Periodic Lotka-Volterra competition equations
}

\author{
J. M. Cushing \\ Department of Mathematics and Program in Applied Mathematics, The University of Arizona, \\ Mathematics Building \#89, Tucson, Arizona 85721, USA
}

\begin{abstract}
The Lotka-Volterra competition equations with periodic coefficients derived from the MacArthur-Levins theory of a one-dimensional resource niche are studied when the parameters are allowed to oscillate periodically in time. Specifically, niche positions and widths, resource availability and resource consumption rates are allowed small amplitude periodicities around a specified mean value. Two opposite cases are studied both analytically and numerically. First only resource consumption rates are allowed to oscillate while niche dimensions and resource availability are held constant. The resulting oscillations in population densities and the strength of the system stability as they depend upon crucial relative phase and amplitude differences between the species' consumption rates are studied. This leads to a clear notion of "temporal niche" and of the effects that such oscillations can have on competitive coexistence. Secondly, all system parameters are allowed to oscillate, although the oscillatory consumption rates are assumed identical for both species. The effects on the population density oscillations and their averages are studied and the "best" choice of the common, periodic resource consumption rate for these two "identical" species competing for similar (even identical) niches is considered.
\end{abstract}

Key words: Lotka-Volterra competition - Periodic coefficients - MacArthurLevins theory - Temporal niche

\section{Introduction}

The vast majority of mathematical models which have been used to describe the growth dynamics of populations is based on autonomous equations. Consequently these models, in which all model parameters and coefficients are constant in time, are really only appropriate for idealized situations in which all biological and environmental conditions do not explicitly change in time. It is widely recognized, on the other hand, that both inherent biological and physical environmental parameters can and in fact often do fluctuate in time and that these fluctuations can have significant effects on the dynamics of the populations [16]. Nonetheless 
it is still true that comparatively very few studies have been made of nonautonomous models with explicit time fluctuating coefficients and that virtually all "fundamental principles" in population dynamics and ecology are theoretically based on autonomous models. A natural question to ask then is to what extent such fundamental principles are affected by time dependent fluctuations in model parameters. In addition it would be of interest to study the specific effects that such fluctuations have on the basic qualitative properties of solutions of specific types of model equations.

In recent years there has indeed appeared a small, but increasing number of papers dealing with these questions. Stochastic fluctuations as well as regular periodic oscillations in model parameters have been investigated for a variety of types of equations for the growth dynamics of various types of populations and interspecies interactions.

With regard specifically to interspecies competition the recognition that periodic environmental factors can significantly effect competitive coexistence was pointed out early in the development of the concept of the ecological niche and the principle of competitive exclusion [10]. The idea that environmental fluctuations, in preventing the attainment of an equilibrium state, can permit the indefinite survival of an otherwise competitively inferior species was studied analytically via Lotka-Volterra dynamics in [11]. General two species LotkaVolterra equations with arbitrary periodic coefficients were studied using bifurcation techniques in [4]. It was shown in [4] that the familiar format of four alternatives for the classical Lotka-Volterra equations (a positive stable or unstable equilibrium or a global attractor on one co-ordinate axis or the other $[16$, p. 179]) whose occurrences depend upon relationships amongst the interaction coefficients as measures of intra- versus interspecies competition, roughly speaking holds up when the coefficients are arbitrary periodic functions of time, provided this format is viewed in an appropriate manner by means of bifurcation diagrams. The periodic Lotka-Volterra equations were further studied in $[13,14]$ with an emphasis placed on the phase differences between the oscillations in the interaction coefficients.

Existence and stability questions for more general periodic, two species competition systems were studied in [7] and for general periodic, $n$-species Kolmogorov systems in [5]. These latter papers deal mainly with the general questions of the mathematical existence and stability of positive periodic solutions, although they do contain some examples which serve to illustrate the fact that periodicities in system parameters can induce unexpected results (such as stable coexistence when averaged coefficients would predict competitive exclusion).

More detailed analyses of some specialized competition systems can be found in [6, 19]. Rosenblatt [19] studies Lotka-Volterra systems under restrictive conditions using perturbation techniques when the periodicities are of small amplitude. This approach allows the derivation of results concerning certain qualitative properties of the periodic solutions. In [6] the author studied in detail a model of non-Lotka-Volterra type for an exploitive (as opposed to an interference) competitive interaction based on Holling II (or Michaelis-Menten) interactive dynamics. This work dramatically shows the significant effects of system parameter 
periodicities in forstalling competitive exclusion under certain conditions, in this case the "time sharing" of a prey resource.

Other papers dealing with certain competition models under the influence of a periodic oscillation in some system parameter include $[1,8,21]$.

The purpose of the present paper is to contribute further to the understanding of the effects on competitive interactions caused by parameter periodicities by describing some analytical and numerical results for the Lotka-Volterra model with coefficients derived from the McArthur-Levins theory of a one-dimensional resource niche. While it is true that Lotka-Volterra dynamics are highly simplistic, as is the MacArthur-Levins theory of an ecological niche, it is nonetheless true that this theory has had a profound impact on ecological thinking, both theoretical and practical, with regard to such fundamental concepts as ecological niche, competitive exclusion, limiting similarity, etc. It seems therefore that despite the simplicity of this model it might be of interest to see what effects that periodic oscillations in the model parameters ... viz. niche width and separation and the resource availability and consumption rates ... might have on these basic concepts and principles.

One can easily make a case for the periodic... or at least roughly periodic oscillation of any one of the basic parameters in the MacArthur-Levins theory. The biological literature is replete with references to periodic fluctuations such as seasonal or daily variations in parameters. Certainly resource availabilities for most animals suffer seasonal variations [17, p. 324]. Seasonal variations in niche utilization or consumption rates has been observed in both animals [15] and in plants [9]. Moreover, resource niche widths can change seasonally [23] as can niche positions on a resource spectrum [17]. Further discussions of these matters can be found in $[17,18]$.

The basic assumption in this paper will be that the coefficients in the classical Lotka-Volterra competition equations are derived from the MacArthur-Levins theory of a one-dimensional resource niche in which the basic parameters are allowed to suffer small amplitude periodic oscillations about a specified mean. Although the assumption that the periodic oscillations are of small amplitude is restrictive it is hoped that the analysis and conclusions here will contribute to at least a preliminary understanding of the effects of oscillations in the parameters of competition models. This assumption is necessary for the perturbation analysis carried out below, but although it prohibits the study of large "catastrophic" fluctuations in model parameters and their effects it is nonetheless true, as pointed out in [19], that to ignore small amplitude oscillations in favor of averaged quantities can be misleading. Moreover, as some of the numerical results below show, the effects of small amplitude oscillations can and often do persist for larger amplitudes. In any case, a study of such small amplitude periodicities certainly contributes to the basic understanding of the effects of model parameter periodicities in general.

After the model equations are set up and discussed in Sect. 2, two roughly opposite cases are analysed in Sect. 3 and Sect. 4 . In Sect. 3 all resource niche and environmental parameters are held constant in time while the two competing species are allowed to have periodically varying resource consumption rates of possibly different phases and relative amplitudes. This case is motivated by an 
interest in what might be termed a "temporal niche" and how it might promote stable coexistence when two species compete for nearly identical (or even exactly identical) resource niches.

In Sect. 4 environmental and resource niche parameters are allowed to fluctuate periodically in time while the two competing species are constrained to having identical resource consumption rates. The goal is to study the effects that such oscillations have on competitive coexistence and on the oscillatory properties of the population densities and to see what strategies such "identical" species might take with regard to their resource consumption rates in order to promote stable coexistence.

Sect. 5 contains a short summary of results and conclusions. An Appendix contains details of some mathematical proofs and derivations.

\section{The model equations}

Based on the MacArthur-Levins theory of a one-dimensional resource niche the coefficients appearing in the Lotka-Volterra system for the densities $P_{1}, P_{2}$ of two competiting species

$$
P_{i}^{\prime}=P_{i}\left(b_{i}-a_{i i} P_{i}-a_{i j} P_{j}\right), \quad 1 \leqslant i \neq j \leqslant 2
$$

are given by $[2,12]$

$$
b_{i}=c_{i} \int_{-\infty}^{+\infty} R(\rho) f_{i}(\rho) d \rho, \quad a_{i j}=c_{i} c_{j} \int_{-\infty}^{+\infty} f_{i}(\rho) f_{j}(\rho) d \rho
$$

Here $R$ is the availability of the resource quantified by the variable $\rho, f_{i}$ is the preference function for the resource by the $i$ th species and $c_{i}$ is the $i$ th species' resource utilization or consumption rate. The most commonly used preference function is Gaussian

$$
f_{i}(\rho)=\left(2 \pi W_{i}^{2}\right)^{-1 / 2} \exp \left(-\left(\rho-D_{i}\right)^{2} / 2 W_{i}^{2}\right)
$$

where $D_{i}$ is referred to as the niche location and $W_{i}$ is the niche width. In the simplest theory it is assumed that the niche widths are identical $W_{i}=W>0$ and that the resource availability is independent of $\rho$ so that $R(\rho) \equiv R>0$. Then it turns out that the coefficients in (2.1) reduce to

$$
b_{i}=c_{i} R, \quad a_{i j}=\left(4 \pi W^{2}\right)^{-1 / 2} c_{i} c_{j} \delta^{|i-j|}, \quad \delta:=\exp \left(-(d / 2 W)^{2}\right)
$$

where $d:=D_{1}-D_{2}$ measures the niche separation.

In the classical case when the system parameters $R, W, c_{i}$ and $d$ are all constant in time, in which case the coefficients $b_{i}$ and $a_{i j}$ are also constants, the equations (2.1) have a unique positive equilibrium

$$
P_{i}=1 /(1+\delta), \quad i=1 \text { and } 2
$$

which is stable (and globally attracting in the positive or first quadrant $P_{i}>0$ ) provided

$$
d \neq 0
$$


i.e. provided the niches are not identical. This is the simplest and most naive theoretical support for the principle of competitive exclusion and limiting similarity, namely that there must be some niche separation in order for the two species competitive interaction to be viable (in the sense that a positive, attracting equilibrium exists). There is however no theoretical limit to how close the niches can be for this two species model, although the stability weakens as $d$ decreases to zero (see Fig. 8). For the case of three or more species, a case not considered here, there is a positive lower bound on $d$ for the existence of a positive stable equilibrium, a fact which is the basis for the celebrated rule-of-thumb that the ratio $d / W$ should be greater than 1 in order to prevent competitive exclusion [12].

This paper deals with the system (2.1) when the model parameters $R, W, c_{i}$ and/or $d$ are periodic functions of time with a common period $p$. In this case the coefficients (2.2) appearing in the Lotka-Volterra system (2.1) are p-periodic in time. Such a nonautonomous system does not in general have an equilibrium solution and the equilibrium stability analysis upon which the fundamental principles mentioned above are based is no longer applicable. In place of a positive equilibrium solution one expects to have a positive $p$-periodic solution and instead of a stability analysis based upon the algebraic theory of eigenvalues one has instead a more difficult analysis based upon Floquet multipliers or exponents.

The results and techniques in $[4,5,7]$ could be applied to these existence and stability questions. However, in this paper a different approach based on regular perturbation techniques is taken in order to gain more specific and detailed results on the qualitative properties of the solutions. For example, of interest will be averaged and extremal values of the population density oscillations, phase relationships and strength of stability as measured by Floquet exponents and how these things depend upon crucial system parameters and their oscillatory properties.

Specifically, it will be assumed that $R, W, c_{i}$ and $d$ are given small amplitude periodicities, relative to specified mean values, of the form

$$
\begin{gathered}
R=R_{0}\left(1+\varepsilon R_{1}(t)\right), \quad W=W_{0}\left(1+\varepsilon W_{1}(t)\right), \quad c_{i}=c_{i 0}\left(1+\varepsilon c_{i 1}(t)\right) \\
R_{0}>0, \quad W_{0}>0, \quad c_{i 0}>0
\end{gathered}
$$

and, because we wish to allow the case of zero average niche separation,

$$
d=d_{0}+\varepsilon d_{1}(t) .
$$

Here $\varepsilon$ is a small real and all periodic perturbations have the same period $p$ and have zero average

$$
a v\left[R_{1}\right]=a v\left[W_{1}\right]=a v\left[c_{i 1}\right]=a v\left[d_{1}\right]=0
$$

where $a v[x]:=p^{-1} \int_{0}^{p} x(t) d t$.

Substituting (2.3)-(2.4) into the coefficients (2.2) one obtains a Lotka-Volterra system (2.1) with $p$-periodic coefficients and with a small parameter $\varepsilon$. When $\varepsilon=0$ the coefficients are constant and the classical equilibrium and stability results described above hold. For $\varepsilon$ sufficiently small one can apply well-known theorems from the theory of differential equations to arrive at the existence and stability of a positive $p$-periodic solution of (2.1) [3]. In addition, for small $\varepsilon$, 
lower order approximations can be in principle routinely calculated by a standard regular perturbation analysis. Since the coefficients (2.2) are analytic in $\varepsilon$ so are the solutions of (2.1) [3]. In particular the positive $p$-periodic solution has an expansion

$$
\begin{aligned}
& P_{i}(t)=\left(1+\delta_{0}\right)^{-1}+\varepsilon y_{i}(t)+\varepsilon^{2} z_{i}(t)+\cdots \\
& \delta_{0}:=\exp \left(-\left(d_{0} / 2 W_{0}\right)^{2}\right)
\end{aligned}
$$

(where the dots will always denote terms higher order than those appearing). To lowest order the oscillatory properties of $P_{i}$ are determined by the coefficient $y_{i}$ (or in some cases by $z_{i}$ ). These coefficients can be computed by the standard procedure of substituting this expansion for $P_{i}$ into the equations (2.1), equating coefficients of like powers of $\varepsilon$ from both sides of the results and solving in recursive fashion the resulting linear nonhomogeneous systems.

Similar techniques can be used to find lower order terms in expansions of the Floquet exponents which are also analytic in $\varepsilon$.

Although straightforward in principal the details of this procedure are extremely tedious and involved. In general it is not even clear that should they be carried out the complicated results would be particularly amenable to analysis and interpretation. In the next two sections this procedure is, however, successfully used for two special cases. First the case of periodic (in fact cosinusoidal) consumption rates $c_{i}$ with all other parameters held constant is considered. Then secondly an opposite case is considered in which the species have identical resource consumption rates and the remaining parameters $R, W$ and $d$ are allowed arbitrary small amplitude periodicities.

\section{Periodic consumptions rates and the temporal sharing of a niche}

The great diversity of means by which species reduce interspecies' competition in order to prevent competitive exclusion can be roughly classified into three categories: the consumption of different resources, the exploitation of different habitats and activity at different times [16, p. 190]. Although the latter means is often thought to occur less frequently in nature than the first two, it is nonetheless widespread and it can be crucial in the viability of some competitive interactions [17, Chap. 13].

In this section the time sharing of a resource niche will be analysed using the Lotka-Volterra-MacArthur-Levins model (2.1)-(2.2) by assuming that the resource consumption rates $c_{i}$ have small amplitude cosinusoidal periodicities while all other model parameters are held constant. In other words, $R_{1} \equiv W_{1} \equiv d_{1} \equiv$ 0 in (2.3) and (2.4) so that

$$
\begin{array}{rlrl}
c_{1} & =c(1+\varepsilon \cos \omega t), & & c_{2}=c(1+\varepsilon a \cos (\omega t+\gamma)) \\
c>0, & 1>\varepsilon>0, & \omega:=2 \pi / p>0, \\
R=R_{0}, & W=W_{0}, & d=d_{0} .
\end{array}
$$

Here $\gamma$ and $a$, satisfying $0 \leqslant \gamma \leqslant \pi$ and $0 \leqslant a \leqslant 1 / \varepsilon$, are the crucial phase and relative amplitude differences in the oscillations of the consumption rates $c_{1}$ and $c_{2}$. 
Note that it is also assumed that both species have the same average consumption rate $c_{i 0}=c$.

The number of parameters appearing in the equations can be reduced by rescaling time from $t$ to $c R_{0} t$ and the population density units from $P_{i}$ to $c P_{i}\left(R_{0}^{2} 4 \pi W_{0}^{2}\right)^{-1 / 2}$. Then the equations to be considered reduce to $(2.1)$ with coefficients

$$
\begin{aligned}
b_{1} & =1+\varepsilon \cos \omega t, \quad b_{2}=1+\varepsilon a \cos (\omega t+\gamma) \\
a_{11} & =(1+\varepsilon \cos \omega t)^{2}, \quad a_{22}=(1+\varepsilon a \cos (\omega t+\gamma))^{2} \\
a_{12} & =a_{21}=\delta(1+\varepsilon \cos \omega t)(1+\varepsilon a \cos (\omega t+\gamma)) \\
\delta & =\delta_{0}=\exp \left(-\left(2 d_{0} / W_{0}\right)^{2}\right)=\exp \left(-(2 d / W)^{2}\right) .
\end{aligned}
$$

Using standard perturbation techniques with the small parameter $\varepsilon$ as described in the previous section, one can (after straightforward but tedious calculations) arrive at the following $\varepsilon$-expansions for the positive $p$-periodic solution of (2.1) with coefficients (3.2):

$$
\begin{aligned}
P_{i}(t) & =\left(1+\delta_{0}\right)^{-1}+\varepsilon y_{i}(t)+\cdots \\
y_{i}(t) & =\frac{1}{2}\left[\left(A_{1}+(-1)^{i-1} A_{2}\right) \cos \omega t+\left(B_{1}+(-1)^{i-1} B_{2}\right) \sin \omega t\right] \\
A_{1} & =-(1+a \cos \gamma+\omega a \sin \gamma) /\left(1+\delta_{0}\right)\left(1+\omega^{2}\right) \\
B_{1} & =(-\omega(1+a \cos \gamma)+a \sin \gamma) /\left(1+\delta_{0}\right)\left(1+\omega^{2}\right) \\
A_{2} & =-\left(\delta_{0}-1\right)\left(\delta_{0}+1\right)^{-2}(\lambda(1-a \cos \gamma)+\varepsilon a \sin \gamma) /\left(\lambda^{2}+\omega^{2}\right) \\
B_{2} & =\left(\delta_{0}-1\right)\left(\delta_{0}+1\right)^{-2}(-\lambda a \sin \gamma+\omega(1-a \cos \gamma)) /\left(\lambda^{2}+\omega^{2}\right) \\
\lambda & =\left(\delta_{0}-1\right) /\left(\delta_{0}+1\right) .
\end{aligned}
$$

The Floquet exponents associated with this solution have the expansions

$$
e_{1}=-1+\cdots, \quad e_{2}=\lambda+\theta \varepsilon^{2}+\cdots .
$$

Since $0<\delta_{0}<1$ and hence $-1<\lambda<0$ it follows that to lowest order in $\varepsilon$ the exponent which determines the strength of the stability of the solution (3.3) is $e_{2}$. Moreover, since $\delta_{0}$ and hence $\lambda$ depend only on the constant niche dimensions $d_{0}$ and $W_{0}$, the effects on the stability of the competitive interaction due to the oscillatory properties of the consumption rates (namely due to the parameters $a$, $\gamma$ and $\omega$ ) are determined by the second-order coefficient $\theta$.

An explicit formula for $\theta$ is very complicated and difficult to get, but inasmuch as the concern here is with two species utilizing a similar (if not identically the same) niches we can use the lowest order term in a $\delta_{0}$-expansion of $\theta$ for $\delta_{0} \sim 1$ (which corresponds to niche separation $d_{0} \sim 0$ ):

$$
\theta=-\omega^{2}\left(a^{2}-2 a \cos \gamma+1\right) / 8\left(1+\omega^{2}\right)+\cdots
$$

where the dots now denote terms of higher order in $\left|\delta_{0}-1\right|$. Furthermore to lowest order in $\left|\delta_{0}-1\right|$ the coefficients $A_{2}$ and $\beta_{2}$ in $y_{i}$ vanish. Thus for similar niches we have the approximate expansions

$$
P_{i}(t) \sim \frac{1}{2}+\left(A_{1} \cos \omega t+B_{1} \sin \omega t\right) \varepsilon+\cdots, \quad e_{2} \sim \theta_{0} \varepsilon^{2}+\cdots
$$


where

$$
\begin{aligned}
& \theta_{0}=-\omega^{2}\left(a^{2}-2 a \cos \gamma+1\right) / 8\left(1+\omega^{2}\right) \\
& A_{1}=-(1+a \cos \gamma+\omega a \sin \gamma) / 2\left(1+\omega^{2}\right) \\
& B_{1}=(-\omega(1+a \cos \gamma)+a \sin \gamma) / 2\left(1+\omega^{2}\right) .
\end{aligned}
$$

These formulas allow for an analysis of the oscillatory properties of the solution $P_{i}$ and the strength of its stability as they depend upon the oscillatory properties of the consumption rates as expressed by the parameters $a, \gamma$ and $\omega$.

(a) The first observation to make is that

$$
\theta_{0}<0 \quad \text { if }(a, \gamma) \neq(1,0) \quad \text { and } \quad \theta_{0}=0 \quad \text { if }(a, \gamma)=(1,0)
$$

In the first case $e_{2}<0$ when $\varepsilon \neq 0$ is small enough (whereas $e_{2}=0$ when $\varepsilon=0$, i.e. in the absence of oscillations in the consumption rates).

Thus we conclude that if there is some difference in the periodic utilization of the resource niche, that is to say, if there is either a nonzero phase difference $(\gamma \neq 0)$ or if there is a difference in amplitudes $(a \neq 1)$, then small amplitude periodicities in the resource consumption rates of the two species result in increased stability.

This conclusion is of course valid only to lowest order in $\varepsilon$. Moreover for fixed $\varepsilon>0$ it may not hold in the case of nearly identical resource consumption rate periodicities $(a, \gamma) \sim(1,0)$, for in this case $\theta_{0} \sim 0$ and higher order terms in the $\varepsilon$-expansion of the Floquet exponent $e_{2}$ can dominate. Indeed the numerical results contained in the graphs of Fig. 1 show in fact that in the case considered there either an increase or a decrease in stability can occur due to consumption rate periodicities depending upon the relationship of the other parameters.

It is interesting and perhaps counterintuitive to find that for the parameter values in Fig. 1(b) the competitive interaction is more stable when the consumption rates are exactly in-phase $(\gamma=0)$ than when they are slightly out-of-phase $(\gamma \sim 0)$. This was typically found to be the case in numerical solutions when the niche separation $d_{0}$ was small.

(b) $\theta_{0}$ and hence stability is maximum when $\gamma=\pi$ (for fixed $a$ and $\omega$ ). Thus, as was undoubtedly intuitively expected, maximal stability occurs when the consumption rates are fully out-of-phase, i.e. when the two species "time share" the niche. Stability is not only maximal in this case but it is considerably stronger than when consumption rates are held constant $(\varepsilon=0)$.

(c) If the resource consumption rates are totally out-of-phase $(\gamma=\pi)$ then $\theta_{0}$ and hence stability is increased if the relative amplitude $a$ is increased. See Fig. 2.

Thus if their resource niches are similar (even identical) two competing species can increase the stability of their interaction and hence minimize the possibility of competitive exclusion by making their temporal utilization of the niches as different as possible. This theoretical result supports the general principle of competitive exclusion in the sense that species risk competitive exclusion when utilizing similar "niches" if one includes temporal utilization dimensions (here $\gamma$ and $a$ ) as well as the resource niche dimensions $d$ and $W$ in the definition of "niche."

(d) If there is some difference in the periodic utilization of the resource (i.e. if $(a, \gamma) \neq(1,0))$ then $\theta_{0}$ and hence stability increases with an increase in the 

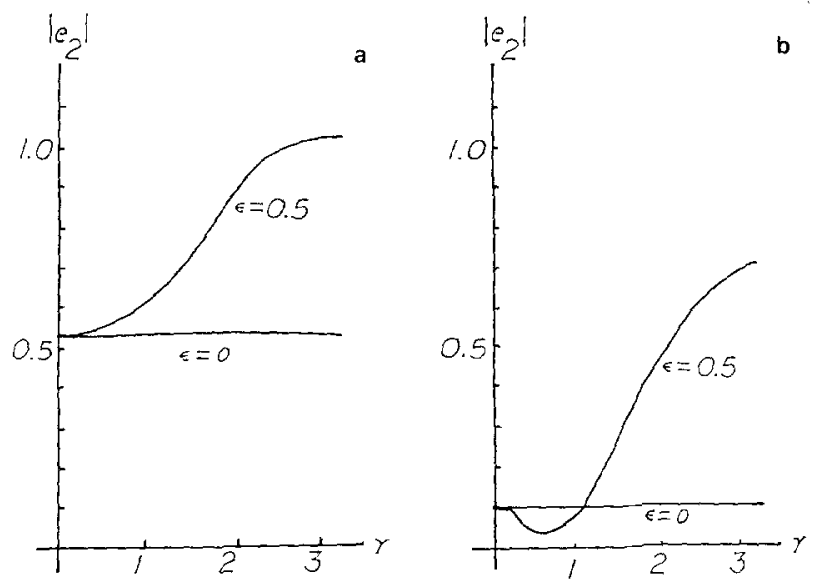

Fig. 1. (a) The unique positive $2 \pi$-periodic solution of the Lotka-Volterra system (2.1) with coefficients (3.2) was computed numerically with parameter values $a=\omega=1.0, \delta=0.9$ (i.e. $d / W=0.6492$ ) and $\varepsilon=0.5$. The magnitude of the stability determining Floquet exponent $e_{2}$, having been calculated numerically from the linearization at the computed $2 \pi$-periodic solution of $(2.1)$, is plotted against the resource consumption rate phase difference $\gamma$. The graph clearly shows maximal stability occurring at $\gamma=\pi$ (totally out-of-phase consumption rates). It is also seen that stability is always greater with periodic consumption rates $(\varepsilon=0.5)$ than with constant consumption rates $(\varepsilon=0)$.

(b) This graph is the same as that in (a) with the same parameter values except that the niches are closer together: $\delta=0.98$ (i.e. $d / W=0.2843$ ). Again maximal stability occurs at $\gamma=\pi$. In this case, however, there is a region $(0<\gamma<1)$ of weakened stability when the consumption rates are only slightly out-of-phase. This is accompanied by a significant drop in the population density levels of $P_{2}$ (see Fig. 5). Such regions were found to be typical when $d / W \sim 0$. The drop in $\mid e_{2} !$ becomes very exaggerated as $d / W \rightarrow 0$
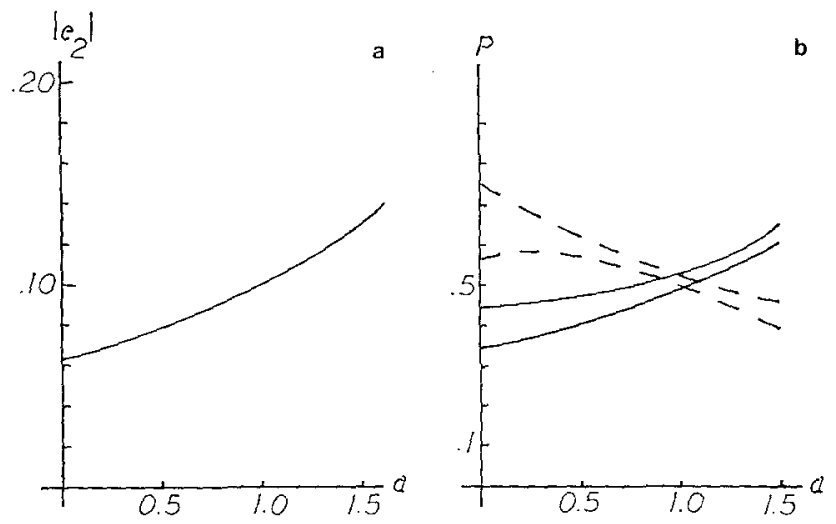

Fig. 2. The graph in (a) shows the predicted increase in stability with an increase in the relative amplitude $a$. Numerical calculations were done with $\omega=1, \delta=0.9$ (i.e. $d / W=0.6492$ ), $\varepsilon=0.5$ and $\gamma=\pi$ in (2.1)-(3.2). In (b) is shown the maxima and minima of the population density oscillations. The dashed lines are for $P_{2}$ and the solid lines for $P_{1}$ 


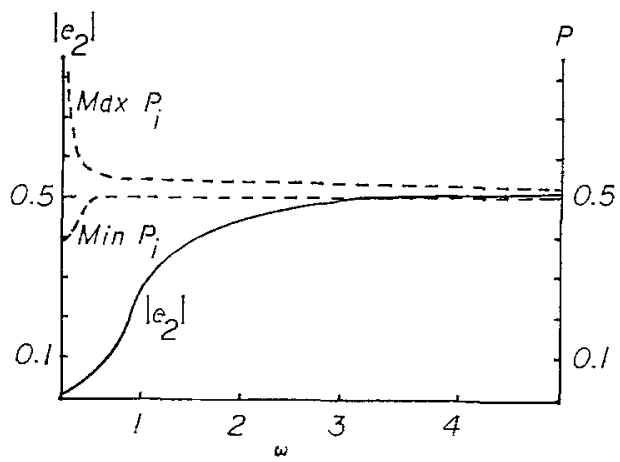

Fig. 3. The magnitude of the stability determining Floquet exponent $e_{2}$ for the linearization of (2.1)-(3.2) at the unique positive $p$ periodic solution for parameter values $a=\varepsilon=$ $1.0, \gamma=\pi$ and $\delta=0.9$ (i.e. $d / W=0.6492$ ) increases with increasing frequency $\omega$ is the consumption rate periodicity. Also seen is an accompanying decrease in the population density oscillation

frequency $\omega$. Changes in strength of stability for extreme values $\omega \sim 0$ and $\omega \sim+\infty$ are however small. The maximum sensitivity of stability to changes in frequency $\omega$ occur near a unique finite value (here $\omega=3^{-1 / 2}$ in the scaled units of time $t$ used above).

Moreover in this case stability is increased with an increase in the amplitude $\varepsilon$ of the periodicities (provided $\varepsilon \sim 0$ ).

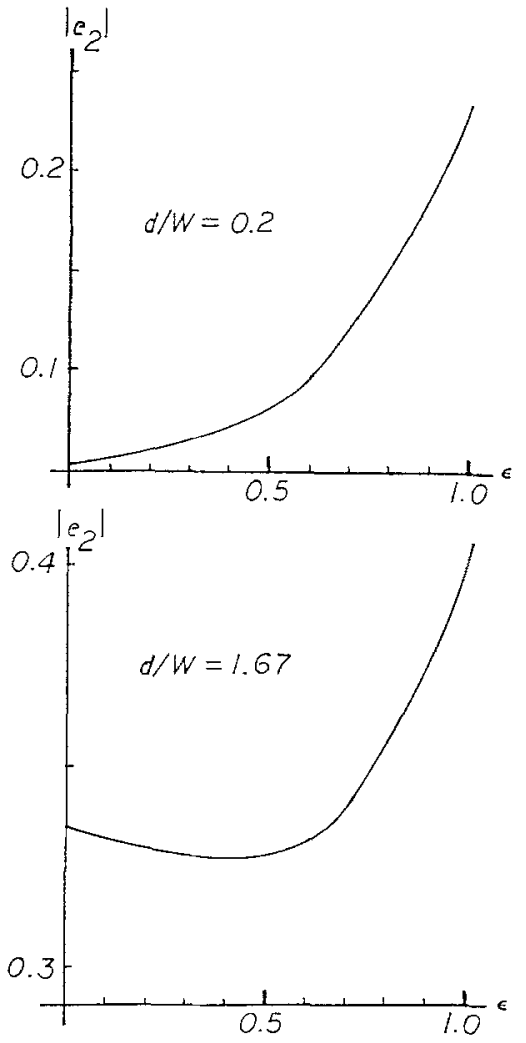

Fig. 4. The magnitude of the stability determining Floquet exponent $e_{2}$ for the linearization of (2.1)-(3.2) at the unique positive $2 \pi$-periodic solution for parameter values $a=\omega=1.0, \gamma=\pi$ is plotted against the consumption rate amplitude $\varepsilon$ for two different niche separation-to-width ratios. Also see Fig. 7 
(e) With regard to the population density oscillations themselves, the amplitude (to lowest order in $\varepsilon$ )

$$
\left(A_{1}^{2}+B_{1}^{2}\right)^{1 / 2}=\frac{1}{4}\left(a^{2}+2 a \cos \gamma+1\right)^{1 / 2}\left(1+\omega^{2}\right)^{-1 / 2}
$$

is minimized for $\gamma=\pi$ ( $a$ and $\omega$ held fixed).

Thus fully out-of-phase resource consumption rates not only maximally enhance stability in the sense of providing the most negative Floquet exponent but also in the sense of providing population density oscillations of minimum amplitude. Minimum amplitude density oscillations presumably help to prevent extinctions due to periodically dangerously low population density levels.

Note that for small $d_{0}$ in Fig. 5(b) one species' population density level drops dangerously low for consumption rates slightly out-of-phase. This is accompanied by a weakened stability as found above (see Fig. 1(b)). These facts were typically found for $d_{0}$ close to zero in numerical integrations and in fact can be very exaggerated for $d_{0}$ made very near or equal to zero.

This suggests that for very similar or identical resource niches competitive exclusion is seriously threatened when consumption rates are slightly out-of-phase. It is better to be in-phase than slightly out-of-phase (but best to be totally out-ofphase). Note that it is $P_{2}$, the species whose consumption rate peaks first (i.e. the species which is the "earliest" in the niche), which is threatened with extinction.

(f) The lowest order Fourier coefficients $A_{1}, B_{1}$ do not simultaneously vanish except when $(a, \gamma)=(1, \pi)$. Thus, unless the resource consumption rates oscillate out-of-phase with equal amplitudes, the population densities oscillate nearly
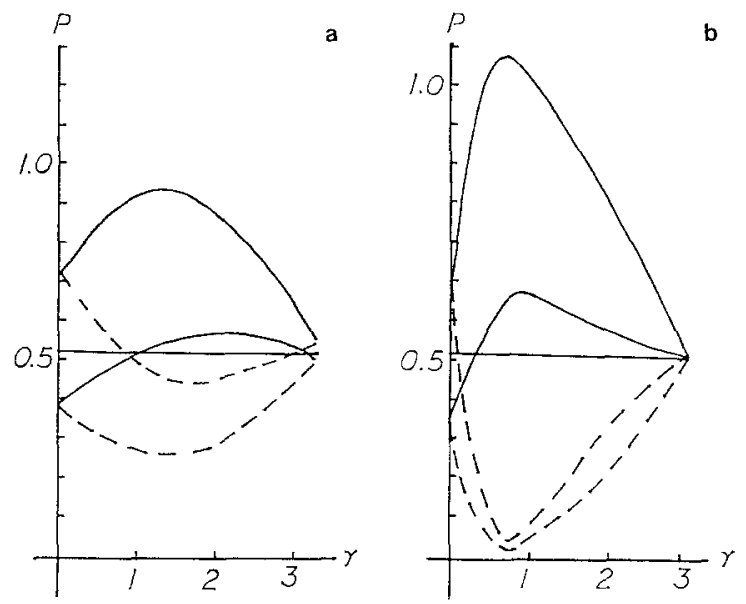

Fig. 5. The maxima and minima of the positive $2 \pi$-periodic solutions $P_{1}$ and $P_{2}$ of $(2.1)-(3.2)$ are plotted against the phase difference $\gamma$ in the resource consumption rates for parameter values $a=\omega=1$ and $\varepsilon=0.5$. (a) is for $\delta=0.90$ (i.e. $d / W=0.6492$ ) and (b) is for $\delta=0.98$ (i.e. $d / W=0.2843$ ). The solid lines are the extrema for $P_{1}$ and the dashed lines are the extrema for $P_{2}$. The Floquet exponent $e_{2}$ for these two cases are plotted in Fig. 1. The horizontal line is the equilibrium value $P_{1}=P_{2}=1 /(1+\delta)$ for the non-oscillatory case $\varepsilon=0$ and is shown for comparison purposes. Note the low level of $P_{2}$ when $\gamma$ is near 1 


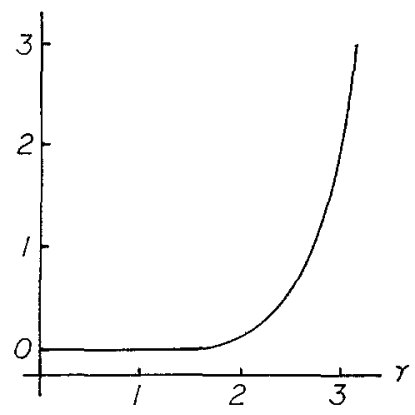

Fig. 6. The phase difference between the components $P_{1}$ and $P_{2}$ of the positive $2 \pi$-periodic solution of (2.1)-(3.2) with $a=\omega=1, \varepsilon=0.5$ and $\delta=0.9$ (i.e. $d / W=0.6492$ ) is plotted against the phase difference $\gamma$ of the resource consumption rates. The components $P_{1}$ and $P_{2}$ are seen to be in-phase until the consumption rates become nearly out-of-phase at which point $P_{1}$ and $P_{2}$ quickly move out-of-phase also
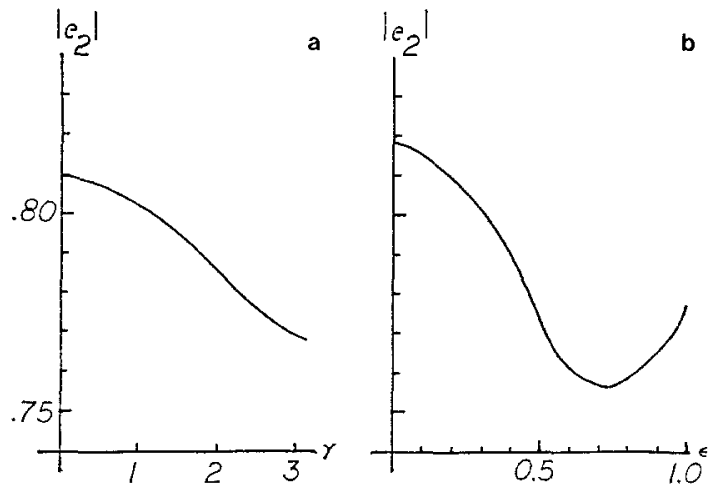

b

Fig. 7. The magnitude of the stability determining Floquet exponent $e_{2}$ of the linearization of (2.1)-(3.2) at the positive $2 \pi$-periodic solution for parameter values $a=\omega=1$ and $\varepsilon=0.1054$ (i.e. $d / W=3.0$ ) is plotted in (a) against the phase difference $\gamma$ in the resource consumption rates (with $\varepsilon=$ 0.5 ) and in (b) against the amplitude $\varepsilon$ (with $\gamma=\pi$ ). Compare these plots for two "widely separated" niches to those in Fig. 1 and Fig. 4 for closer niches

in-phase. On the other hand, the numerical results in Fig. 6 show that when $(a, \gamma) \sim(1, \pi)$ the densities oscillate nearly out-of-phase.

Any of the above conclusions can radically alter if the niches are far apart $\delta \ll 1$ or if the amplitude $\varepsilon>0$ is large or if the temporal niches are nearly identical $(a, \gamma) \sim(1,0)$.

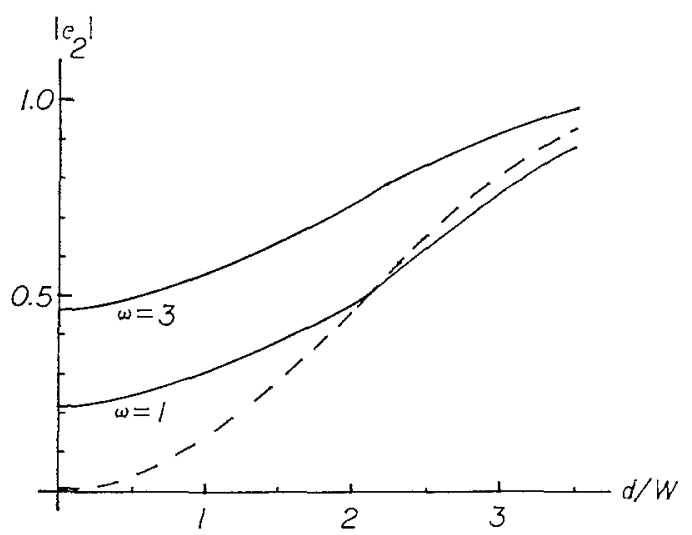

Fig. 8. The magnitude of the stability determining Floquet exponent $e_{2}$ of the linearization of (2.1)-(3.2) at the positive $p$-periodic solution for $a=\varepsilon=1$ and $\gamma=$ $\pi$ is plotted against the niche separation to width ratio $d / W$. The dashed line is the magnitude of the stability determining eigenvalue of the equilibrium of the classical autonomous case of nonoscillatory parameters $(\varepsilon=0)$ 
For example in Fig. 7 where the niche separation is relatively large maximal stability does not occur at $\gamma=\pi$ but on the contrary at $\gamma=0$. In that example it is more advantageous for the competing species to have in-phase rather than out-of-phase resource consumption rates.

Figure 7(b) shows the relationship of the strength of stability to the amplitude $\varepsilon$. Out-of-phase resource consumption rates are stabilizing for similar niches, but are destabilizing for niches sufficiently separated. This is further illustrated in Fig. 8 in which strength of stability is plotted against niche separation (or more precisely the niche separation to width ratio) and is compared to the classical autonomous case of no periodicities $\varepsilon=0[2,12]$.

\section{Periodic niche dimensions}

In the preceding section all environmental and resource niche parameters $R, d$ and $W$ were held constant in time while the effects of periodic resource consumption rates were studied. In this section an opposite case will be considered in which $R, d$ and $W$ are allowed to oscillate in an arbitrary periodic manner while the resource consumption rates, although not necessarily restricted to be constant in time, will be required to be identical for both species $c:=c_{1}=c_{2}$. The situation then is that in which two identical, competing species (identical in the sense that they have identical resource consumption rates) find themselves in a periodic environment. The goal is to see what effects these environmental periodicities have on the stability of the competitive interaction and on the resulting population density oscillations. We will also see what "best" resource consumption rate $c$ the two species might adopt in order to enhance their mutual coexistence (i.e. to avoid competitive exclusion).

When $c_{1}=c_{2}=c$ in the MacArthur-Levins coefficients (2.2) the Lotka-Volterra system of equations (2.1) becomes symmetric

$$
P_{i}^{\prime}=P_{i}\left(c R-c^{2}\left(4 \pi W^{2}\right)^{-1 / 2} P_{i}-\delta c^{2}\left(4 \pi W^{2}\right)^{-1 / 2} P_{j}\right), \quad 1 \leqslant i \neq j \leqslant 2 .
$$

This system (which is different from the symmetric system studied by Rosenblat in [19]) has the form of that studied in the appendix. The mathematical results there imply that this system of equations has a unique, globally attracting positive $p$-periodic solution and that this solution has the form $P_{1}=P_{2}=P$ where $P$ is the unique positive $p$-periodic solution of the periodic logistic equation

$$
P^{\prime}=r P(1-P / K)
$$

where

$$
r:=c R, \quad K:=R\left(4 \pi W^{2}\right)^{1 / 2} / c(1+\delta) .
$$

Furthermore the Floquet exponents are given by

$$
e_{1}=-p \text { av }[c R], \quad e_{2}=-p \text { av }\left[c^{2} P(1-\delta)\left(4 \pi W^{2}\right)^{-1 / 2}\right] .
$$

Suppose now that the system parameters are subjected to small amplitude periodicities of the form $(2.3)-(2.4)$ with

$$
\begin{aligned}
c_{1}(t) & \equiv c_{2}(t) \equiv c(t):=c_{0}(1+\varepsilon w(t)) \\
c_{0} & >0, \quad \operatorname{av}[w]=0 .
\end{aligned}
$$


Then the logistic coefficients (4.2) have $\varepsilon$-expansions

$$
\begin{aligned}
r(t)= & r_{0}+\varepsilon r_{1}(t)+\cdots, \quad K(t)=K_{0}+\varepsilon K_{1}(t)+\varepsilon^{2} K_{2}(t)+\cdots \\
r_{0}:= & c_{0} R_{0}, \quad r_{1}:=c_{0} R_{0}\left(w+R_{1}\right), \quad K_{0}:=R_{0}\left(4 \pi W_{0}^{2}\right)^{1 / 2} / c_{0}\left(1+\delta_{0}\right) \\
K_{1}:= & K_{0}\left(W_{1}+R_{1}-w-\delta_{1}\left(1+\delta_{0}\right)^{-1}\right) \\
K_{2}:= & K_{0}\left(R_{1} W_{1}-\left(W_{1}+R_{1}\right)\left(w+\delta_{1}\left(1+\delta_{0}\right)^{-1}\right)+w^{2}\right. \\
& \left.+\left(w \delta_{1}-\delta_{2}\right)\left(1+\delta_{0}\right)^{-1}+\delta_{1}^{2}\left(1+\delta_{0}\right)^{-2}\right)
\end{aligned}
$$

where

$$
\begin{gathered}
\delta=\delta_{0}+\varepsilon \delta_{1}+\varepsilon^{2} \delta_{2}+\cdots \\
\delta_{0}:=\exp \left(-\left(d_{0} / 2 W_{0}\right)^{2}\right), \quad \delta_{1}:=\delta_{0} d_{0}^{2}\left(W_{1}-d_{1} / d_{0}\right) / 2 W_{0}^{2}
\end{gathered}
$$

Standard regular perturbation procedures yield, from the coefficients (4.4), a solution

$$
P(t)=K_{0}\left(1+\varepsilon x_{1}(t)+\varepsilon^{2} x_{2}(t)+\cdots\right)
$$

of the logistic (4.1). The coefficients $x_{1}, x_{2}, \ldots$ are found by substitution into (4.1) and a solving of the linear equations obtained by equating coefficients of like powers of $\varepsilon$ from both sides.

The Floquet exponents (4.3) have $\varepsilon$-expansions

$$
\begin{aligned}
& e_{1}=-p c_{0} R_{0}-p \operatorname{av}\left[w R_{1}\right] \varepsilon^{2}+\cdots \\
& e_{2}=-p K_{0} c_{0}^{2}\left(1-\delta_{0}\right)\left(4 \pi W_{0}^{2}\right)^{-1 / 2}+\psi \varepsilon^{2}+\cdots
\end{aligned}
$$

for a coefficient $\psi$ yet to be calculated.

Our concern here will be with the case $d_{0} \sim 0$, i.e. with the case when the niches are close together in the sense that the average niche separation is zero. In this case the stability determining exponents, i.e. the one with the smallest magnitude, is clearly $e_{2}$ since $\delta_{0} \sim 1$ and $\varepsilon \sim 0$.

If $\psi_{0}$ denotes the coefficient $\psi$ in $e_{2}$ when $d_{0}=0$, then to lowest order the unique positive periodic solution is stabilized if $\psi_{0}<0$ and is destabilized if $\psi_{0}>0$. This coefficient turns out to be

$$
\psi_{0}=-c_{0} R_{0} \operatorname{av}\left[d_{1}^{2}(t)\right] / 8 W_{0}^{2}
$$

which satisfies $\psi_{0}<0$ if $d_{1} \neq 0$.

We see then that this analysis implies the reasonable result that any periodicity in niche separation of niches which on average are identical enhances stability. Moreover, the analysis shows that to lowest order, periodic oscillations in niche separation dominate system stability in that the oscillatory terms $W_{1}, w$ and $R_{1}$ do not appear in $\psi_{0}$.

On the other hand, periodic oscillations in $W, w$ and/or $R$ do have an effect on the oscillatory properties of the population densities. The coefficients $x_{i}$ in the solution (4.5) are given by the unique periodic solutions of the linear nonhomogeneous equations

$$
\begin{aligned}
& x_{1}^{\prime}+r_{0} x_{1}=r_{0} K_{1} \\
& x_{2}^{\prime}+r_{0} x_{2}=r_{0} K_{2}-r_{0}\left(K_{1}-x_{1}\right)^{2}+r_{0} r_{1}\left(K_{1}-x_{1}\right)
\end{aligned}
$$


where for $d_{0}=0$ (and hence $\delta_{0}=1, \delta_{1}=0$ and $\left.\delta_{2}=-\left(d_{1} / 2 W_{0}\right)^{2}\right)$

$$
\begin{aligned}
& K_{1}=R_{0} W_{0} \pi^{1 / 2}\left(W_{1}+R_{1}-w\right) / c_{0} \\
& K_{2}=R_{0} W_{0} \pi^{1 / 2}\left(R_{1} W_{1}-\left(W_{1}+R_{1}\right) w+w^{2}-\frac{1}{2} \delta_{2}\right) / c_{0}
\end{aligned}
$$

The amplitude and phase of $x_{1}$, and hence of $P$ to lowest order, is determined through Eq. (4.7) by $K_{1}$. The phase of $x_{1}$ is consequently never exactly that of $K_{1}$ (i.e. of $K$ to lowest order), although it approaches that of $K_{1}$ for large $r_{0}$. The amplitude of $x_{1}$ is always less than that of $K_{1}$ also. If we take as the "most stable" competitive interaction the one with minimum amplitude oscillations in population densities, then the best choice for the two species is to consume the resource periodically according to the formula

$$
w=R_{1}+W_{1} \text {. }
$$

Then $K_{1}$ and hence $x_{1}$ vanish identically and $P=K_{0}\left(1+\varepsilon^{2} x_{2}+\cdots\right)$ where

$$
\begin{gathered}
x_{2}^{\prime}+r_{0} x_{2}=r_{0} K_{2} \\
K_{2}=R_{0} W_{0} \pi^{1 / 2}\left(R_{1} W_{1}+d_{1}^{2} / 8 W_{0}\right) / c_{0} .
\end{gathered}
$$

With regard to the average population density we see that $\operatorname{av}\left[K_{1}\right]=0$ and consequently av $\left[x_{1}\right]=0$. Thus there is a second order change in average population density due to parameter periodicities. Under the condition (4.8) this change in average is determined by $K_{2}$ :

$$
\operatorname{av}\left[x_{2}\right]=\operatorname{av}\left[K_{2}\right]=R_{0} W_{0} \pi^{1 / 2}\left(\operatorname{av}\left[R_{1} W_{1}\right]+\operatorname{av}\left[d_{1}^{2}\right] / 8 W_{0}\right) / c_{0} .
$$

In conclusion, a competitive interaction of two identical species $\left(c_{1} \equiv c_{2}\right)$ competing for identical averaged niches $\left(d_{0}=0\right)$ is made more stable by any periodic oscillation in niche separation and the resulting population density oscillations are maximally damped in amplitude if the resource consumption rate is periodic and is taken to be the sum of the periodicities in the resource availability and the niche width (4.8). In this case an increased average population density is attained by having a periodic oscillation in niche width which is in-phase with the periodic oscillation in resource availability $\left(\operatorname{av}\left[R_{1} W_{1}\right]>0\right)$. Furthermore, the periodic oscillation in niche separation not only increases stability but also the average population density ( $\operatorname{av}\left[d_{1}^{2}\right]>0$ hence $\operatorname{av}\left[x_{2}\right]>0$ ).

These conclusions are illustrated by the results of numerical integrations graphed in Figs. 9-12. These graphs were drawn from many integrations of the periodic logistic (4.1)-(4.2) with the assigned periodicities

$$
\begin{aligned}
w & =r_{c} \cos \left(\omega t+\gamma_{c}\right), & W_{1} & =r_{W} \cos \left(\omega t+\gamma_{W}\right) \\
R_{1} & =r_{R} \cos \left(\omega t+\gamma_{R}\right), & d_{1} & =r_{d} \cos \omega t .
\end{aligned}
$$

Finally consider briefly the case of nearly zero average, but a nonoscillatory niche separation $d_{0} \sim 0, d_{1} \equiv 0$. In this case the coefficient $\psi$ in the stability determining Floquet exponent (4.6) vanishes for $d_{0}=0$ and hence in order to determine the effects on stability of the periodicities in the system parameters the first nonzero term in a $d_{0}$-expansion of $\psi$ must be calculated. If again (4.8) is assumed to hold (so that the amplitude of the population density oscillations is minimized), computational simplicity results and it turns out that

$$
\psi=-d_{0}^{2} R_{0} c_{0} \text { av }\left[W_{1}^{2}-3 R_{1} W_{1}+R_{1}^{2}\right] / 8 W_{0}^{2}+\cdots
$$




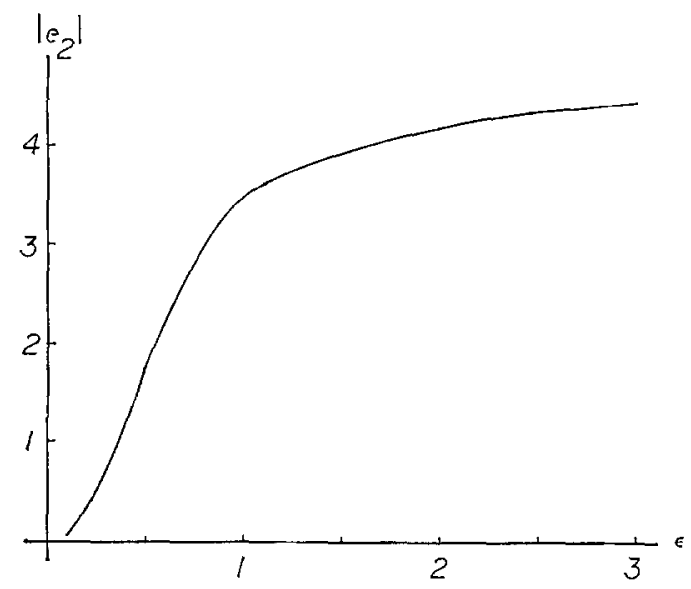

Fig. 9. The magnitude of the stability determining Floquet exponent $e_{2}$ of the system (2.1) with coefficients (2.2)-(2.3) under the cosinusoidal periodicities (4.11) is plotted against the amplitude $\varepsilon$. Here the average niche separation $d_{0}=$ 0 (with $r_{d}=1$ ) and the niche dimensions are nonoscillatory $r_{W}=r_{R}=0$ with averages $\quad W_{0}=0.2, \quad R_{0}=5.0 . \quad$ The resource consumption rate is also constant $c_{0}=1.0, r_{\mathrm{c}}=0$. The period is $2 \pi$ $(\omega=1)$. This graph shows a strengthened stability with increased amplitude in the niche separation periodicity

where the dots denote terms of order $d_{0}^{3}$. Stability is enhanced by the periodic parameter oscillations in this case if

$$
\operatorname{av}\left[W_{1}^{2}-3 R_{1} W_{1}+R_{1}^{2}\right]>0
$$

whereas stability is weakened if the opposite inequality holds.

For example, stability is enhanced if only one of the two parameters $R$ or $W$ oscillates (i.e. if $W_{1} \equiv 0, R_{1} \neq \equiv 0$ or vice versa). "Enhanced" means here that stability is stronger ( $e_{2}$ is more negative) in the presence of the periodicities $(\varepsilon>0)$ than without periodicities $(\varepsilon=0)$.

Very roughly speaking (4.12) implies that an out-of-phase relationship ( $\operatorname{av}\left[R_{1} W_{1}\right]<0$ or a large relative amplitude difference) between $R$ and $W$ enhances system stability while an in-phase relationship weakens stability.

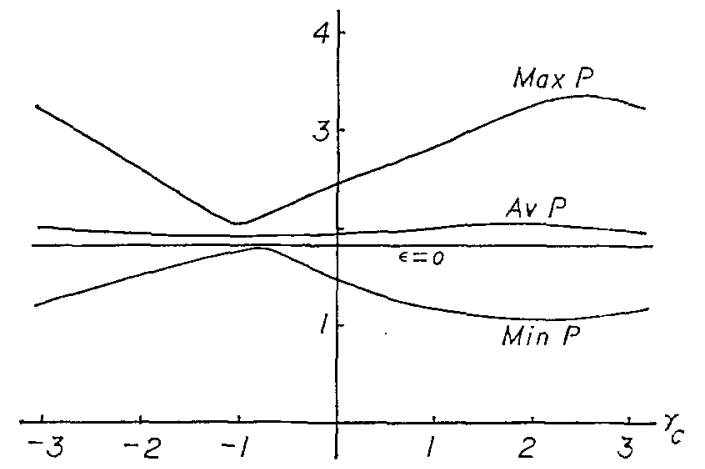

Fig. 10. The extrema of the positive $2 \pi$ periodic solution $P_{1}=P_{2}=P$ of (2.1) with coefficients (2.2)-(2.3) under the cosinusoidal periodicities (4.11), $\omega=1$, are plotted against the consumption rate phase $\gamma_{c}$. Here $d_{0}=0, r_{d}=1, W_{0}=0.2$, $r_{W}=1$ and $R_{0}=5, r_{R}=1$ with $\varepsilon=0.2$. The phases are (relative to the cosine oscillation of the niche separation $d_{1}=$ $\cos t) \gamma_{W}=0$ and $\gamma_{R}=-\pi / 2$. Note that the minimum oscillation in population density $P$ occurs very near the theoretically predicted consumption rate phase $\gamma_{c}=-\pi / 4$ when $w=R_{1}+W_{1}$. The equilibrium density when no oscillations are present $(\varepsilon=0)$ is shown for comparison 


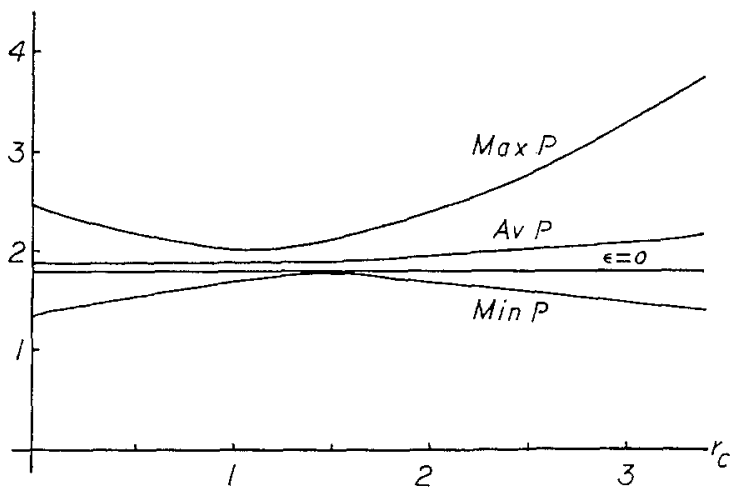

Fig. 11. The extrema of the positive $2 \pi$-periodic solution $P_{1}=P_{2}=P$ of $(2.1)$ are shown for the same coefficients as in Fig. 10 except that $\gamma_{c}=-\pi / 4$ while the relative amplitude $r_{c}$ of the consumption rate is varied. The minimum amplitude of the population density oscillation occurs very near the theoretically predicted value $r_{c}=2^{1 / 2}$ where $w=W_{1}+R_{1}$. Note again that the average density is greater than the equilibrium density when no oscillations occur $(\varepsilon=0)$.

Moreover $P=K_{0}\left(1+\varepsilon^{2} x_{2}+\cdots\right)$ where $x_{2}$ satisfies (4.9) with $K_{2}$ given by (4.10) with $d_{1} \equiv 0$. Thus

$$
\operatorname{av}\left[x_{2}\right]=R_{0} W_{0} \pi^{1 / 2} \operatorname{av}\left[R_{1} W_{1}\right] / c_{0}
$$

and an increase in average population density results if $\operatorname{av}\left[R_{1} W_{1}\right]>0$.

In summary, if two identical species $\left(c_{1} \equiv c_{2}\right)$ share nearly identical niches $\left(d_{0} \sim 0\right)$ whose separation does not oscillate $\left(d_{1} \equiv 0\right)$ and they do so with a periodic consumption rate (4.8) selected to minimize the amplitude of their population density oscillations, then the effects on stability and on the average population density are determined by the phase relationship and by the relative amplitudes of the periodicities in resource availability $R$ and niche width $W$. The effects on stability and on average population density are opposite: that which increases stability (i.e. an out-of-phase relationship) decreases average population density and vice versa.

These conclusions are illustrated in Figs. 12-14.

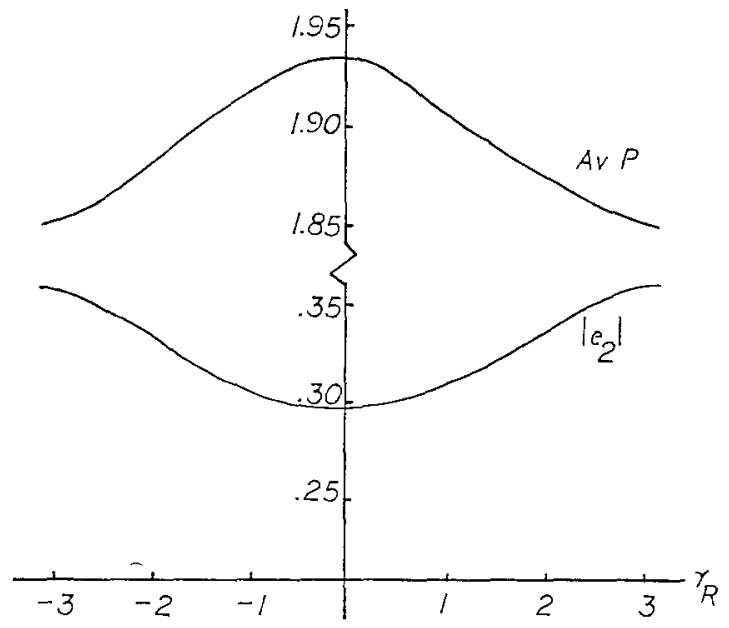

Fig. 12. As in Figs. 10 and $11, \varepsilon=0.2$, $d_{0}=0, r_{d}=r_{W}=r_{R}=1, W_{0}=0.2, c_{0}=$ 1 and $R_{0}=0.5$. Here however $\gamma_{W}=0$ and the consumption rate oscillation is chosen so that $w=R_{1}+W_{1}$. Thus $r_{c}=\left(2\left(1+\cos \gamma_{R}\right)\right)^{1 / 2}$ and $\gamma_{c}=$ $\operatorname{Tan}^{-1} \sin \gamma_{R} /\left(1+\cos \gamma_{R}\right)$. The average population density is maximized when $R$ and $W$ are in-phase $\left(\gamma_{R}=\right.$ 0 ) at which point, however, stability is minimized 


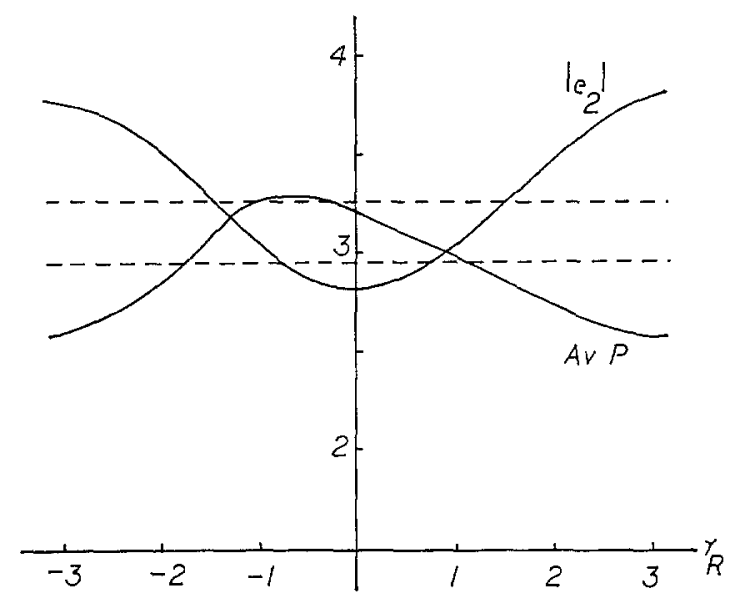

Fig. 13. Unlike in Figs. $9-12$ the average niche separation here is not zero but $d_{0}=0.5$. Also the niche separation is constant in time $r_{d}=0$. Other parameter values in the oscillations (4.11) are $r_{W}=r_{R}=1$, $\gamma_{W}=0$ and $w=R_{1}+W_{1}$, i.e. $r_{c}=\left(2\left(1+\cos \gamma_{R}\right)\right)^{1 / 2}$ and $\gamma_{c}=\operatorname{Tan}^{-1} \sin \gamma_{R} /\left(1+\cos \gamma_{R}\right)$. The parameter averages are $W_{0}=0.2, R_{0}=5.0$ and $c_{0}=1.0$. The amplitude is $\varepsilon=0.5$. As theoretically predicted the graphs show an inverse relationship between the average density and the system stability with maximum stability occurring roughly when $R_{1}$ and $W_{1}$ are out-of-phase $\left(\gamma_{R}=\pi\right)$. The upper dashed line shows the nonoscillatory $(\varepsilon=0)$ eigenvalue for comparison to the Floquet exponent while the lower dashed line shows the equilibrium density when $\varepsilon=0$ for comparison with the average oscillatory density

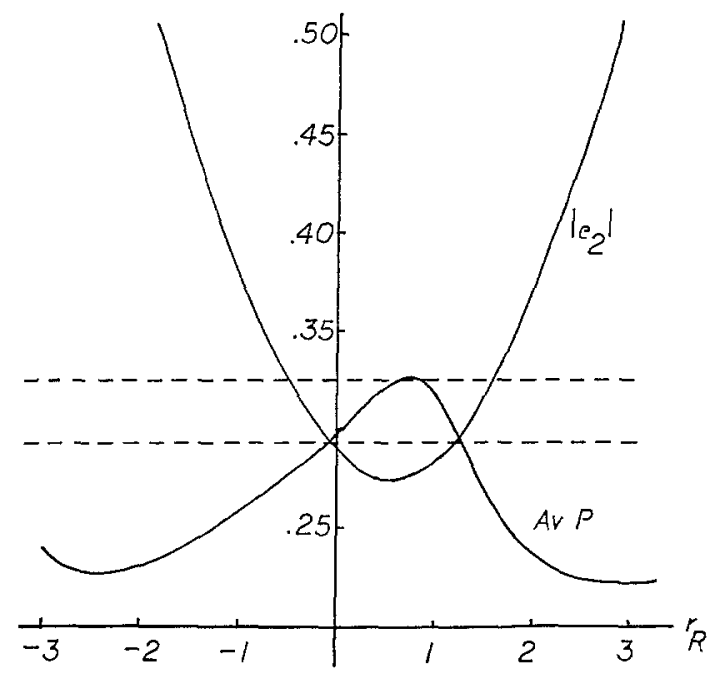

Fig. 14. As in Fig. 13 the niche separation is held constant $d_{0}=0.5, r_{d}=0$ and the oscillation in the consumption rate is chosen so that $w=R_{1}+W_{1}$. Parameter values are $\varepsilon=0.5, W_{0}=0.2$, $r_{W}=1, \gamma_{W}=0, R_{0}=5, \gamma_{R}=0$ and $c_{0}=$ $1, r_{c}=1+r_{R}, \gamma_{c}=0$. Here $R_{1}$ and $W_{1}$ have relative amplitudes $r_{R}$ and are in-phase when $r_{R}>0$ and out-of-phase when $r_{R}<0$. The dashed lines are the equilibrium levels when $\varepsilon=0$. The condition (4.12) predicts enhanced stability when $\varepsilon \neq 0$ when $\left|r_{R}\right|>2^{1 / 2}$, which is roughly true in the graph. The analysis predicts a decreased average density for $r_{R}<0$ and an increased average for $r_{R}>0$, which holds true in the graph except for "large" relative amplitudes $r_{R}>1$

\section{Concluding remarks}

The classical Lotka-Volterra equations were used in this paper to study the effects that time periodic oscillations in model parameters have on a two-species competitive interaction. The coefficients were taken to be those of the simplest case from the MacArthur-Levins theory of a one-dimensional resource niche. Thus, 
Gaussian resource preference functions were used with equal standard deviations or "niche widths" $W$ and the resource availability $R$ was assumed independent of resource type. If either $W$ or $R$ or if either the niche separation $d$ or the resource consumption rates $c_{i}$ are allowed to fluctuate periodically in time, then the corresponding Lotka-Volterra system has time periodic coefficients. As a result the classical equilibrium analysis and its results are no longer applicable.

In place of the fundamental questions concerning the existence and stability of positive equilibria, one now has the mathematically more difficult questions concerning the existence and stability of positive periodic solutions. Recent general theorems and techniques $[4,5,7]$ can be applied to these fundamental questions or, in the case considered here of small amplitude periodicities, standard small parameter perturbation techniques also yield existence and stability. In the latter case, these regular perturbation techniques can also yield specific results concerning the properties of the solution and its stability as they depend on the properties of the periodicities in the model parameters. In Sects. 3 and 4 such an analysis is carried out for two restricted and roughly opposite cases.

First, if species consumption rates oscillate periodically (in Sect. 3 cosinusoidally) while all other system parameters are held constant in time, it was found that the stability of the competitive interaction for two species competing for an identical (or nearly identical) resource niche is always increased, at least when there is some difference in their "temporal niche," i.e. if either there is a phase or a relative amplitude difference in their resource consumption rates. For any relative amplitude difference maximal stability occurs when the consumption rates are totally out-of-phase, as might be expected.

What is perhaps unexpected is that minimal stability does not always occur when the consumption rates are exactly in-phase, but for very similar niches (average separation nearly zero) typically occurs for consumption rates slightly out-of-phase. In addition, in this situation the species whose consumption rate peaks earliest is threatened with drastically low population density levels (maximum and minimum).

This latter result might have implications with regard to the possible evolution of temporal niche sharing. For, if two similar species sharing a niche in-phase attempt to avoid competitive exclusion by adopting a time sharing out-of-phase resource utilization strategy this could not be done by small adaptive changes in the phase difference without first passing through a slightly out-of-phase stage which would be even more threatening to coexistence. Or, put another way, if one species wishes to eliminate its competitor it should put its consumption rate slightly out-of-phase with that of its competitor (and, in the case considered here, so that it peaks later).

Other properties of the population density oscillations and their stability are also studied in Sect. 3. In particular it is pointed out that the conclusions reached above may be drastically altered or even reversed without the basic assumption that the parameter periodicities are of small amplitude or if there is no difference in the temporal niches. For example, if the niche separation is large enough then it is best not to have periodic consumption rates, but to hold consumption rates constant in time or if periodic consumption rates are required then it is more stabilizing to be in-phase rather than out-of-phase. 
Secondly, the case of periodic environmental and niche oscillations was considered in Sect. 4 under the assumption that the species are identical in the sense that they have the same resource consumption rates and that they are competing for an identical (or nearly identical) niche. It was found that in this case system stability is dominated to lowest order by the periodicities in niche separation in that any such oscillation is stabilizing while any periodicity in any other system parameter has no effect (to the same order). Moreover, when there is a periodic oscillation in niche separation a further stabilization can be attained by choosing the common species resource consumption rate to be oscillatory with a periodicity which is a sum of those in the resource availability and the niche width. This has the effect of minimizing the amplitude of the oscillations in population densities.

On the other hand should the niche separation not oscillate in time the situation becomes a good deal more complicated. The species can still minimize the amplitude of their density oscillations by choosing their resource consumption rates as a sum of the periodicities in niche width and resource availability, but the strength of the system stability as measured by Floquet exponents depends in a non-straightforward way on the relative phase and amplitude differences between these two periodic environmental parameters. It can be said nonetheless that the analysis roughly indicates that an out-of-phase relationship between niche width and resource availability is stabilizing while an in-phase relationship is destablizing. There is, however, a trade-off in that an out-of-phase relationship leads to lowered average population densities (while an in-phase relationship leads to higher averages).

We have seen that the simplest classical model of two species competition, the Lotka-Volterra system (2.1), becomes nontrivial to analyse if the coefficients are allowed to be periodic in time. Even the simplest form for these coefficients derived from the MacArthur-Levins theory of a one-dimensional resource niche leads to analytically intractable equations without further simplifying assumptions. It is hoped that the analysis of the two simplified special cases studied here and the results obtained from it contribute in some way to the fundamental understanding of the effects that time periodic oscillations in system parameters can have on the basic theory of competing species.

\section{Appendix}

Consider the symmetric system

$$
P_{i}^{\prime}=P_{i}\left(\alpha(t)-\beta(t) P_{i}-\xi(t) P_{j}\right), \quad 1 \leqslant i \neq j \leqslant 2
$$

where $\alpha, \beta$ and $\xi$ are all positive periodic, continuous functions of a common period $p$. Associated with this system is the periodic logistic equation

$$
P^{\prime}=P(\alpha(t)-(\beta(t)+\xi(t)) P) .
$$

Note that a solution $P$ of (A.2) either never vanishes or is identically zero. Similarly each component $P_{i}$ of a solution pair of (A.1) either never vanishes or is identically zero.

Theorem 1. (i) If $P$ solves (A.2) then $P_{1} \equiv P_{2} \equiv P$ solve $(A .1)$.

(ii) Assume that $\xi(t) \leqslant \beta(t)$ for all $t$, but $\xi \neq \beta$. If $P_{1}, P_{2}$ are positive periodic solutions of $(A .1)$ then $P_{1} \equiv P_{2}$ and $P \equiv P_{1}$ solves (A.2). 
Proof. Part (i) is trivial. To prove (ii) let $P_{1}, P_{2}>0$ be periodic solutions of (A.1). If the equation $i=1$ in (A.1) is divided by $P_{1}$ and the result averaged over one period, then one gets (because $P_{1}$ is periodic) the result

$$
\operatorname{av}[\alpha]=\operatorname{av}\left[\beta P_{1}+\xi P_{2}\right] .
$$

From this equality and the assumption $\xi \leqslant \beta(\xi \neq \beta)$ it follows that

$$
\operatorname{av}[\alpha]<\operatorname{av}\left[\beta\left(P_{1}+P_{2}\right)\right] .
$$

Let $x=P_{1}-P_{2}$ and subtract the two equations in (A.1). This leads to

$$
x^{\prime}=(\alpha(t)-\tilde{\beta}(t)) x, \quad \tilde{\beta}:=\beta\left(P_{1}+P_{2}\right)
$$

It follows that either $x \equiv 0$ or $x$ is never zero. In the latter case, one can divide (A.4) by $x$ and average the result to obtain (because $x$ is periodic)

$$
\operatorname{av}[\alpha]=\operatorname{av}[\tilde{\beta}]=\operatorname{av}\left[\beta\left(P_{1}+P_{2}\right)\right],
$$

which is a contradiction to (A.3). Thus it must be the case that $x \equiv 0$ or in other words that $P_{1} \equiv P_{2}$. That $P \equiv P_{1}$ then solves (A.2) is trivial.

The periodic logistic (A.2) can be integrated in closed form and it is rather easy to show that it has a unique positive periodic solution [20]. This fact yields the following result for (A.1).

Corollary 1. System (A.1) has at least one positive periodic solution. If $\xi(t) \leqslant \beta(t)$ for all $t$, but $\xi \neq \equiv$, then (A.1) has a unique positive periodic solution. This solution satisfies $P_{1} \equiv P_{2}$ where $P_{1}$ and $P_{2}$ satisfy (A.2).

With regard to the stability of the positive periodic solution we have the following result.

Corollary 2. If $\xi(t) \leqslant \beta(t)$ for all $t$, but $\xi \not \equiv \beta$, then the unique positive periodic solution of $(A .1)$ is globally attracting (in the positive quadrant).

Proof. Theorem 1 and the facts discussed above concerning the periodic logistic (A.2) imply that (A.1) has exactly four nonnegative solutions:

$$
P_{1} \equiv P_{2} \equiv 0 \quad \text { and } \quad P_{1} \equiv 0, \quad P_{2} \equiv \tilde{P} \quad \text { and } \quad P_{1} \equiv \tilde{P}, \quad P_{2} \equiv 0 \quad \text { and } \quad P_{1} \equiv P_{2} \equiv P
$$

where $P>0, \tilde{P}>0$ are the unique positive periodic solutions of the periodic logistic (A.2) and

$$
\tilde{P}^{\prime}=\tilde{P}(\alpha-\beta \tilde{P})
$$

respectively.

The null solution $P_{1} \equiv P_{2} \equiv 0$ is clearly unstable since the linearized Floquet exponents are both av $[\alpha]>0$.

The axis solutions $P_{1} \equiv 0, P_{2} \equiv \tilde{P}$ and $P_{1} \equiv \tilde{P}, P_{2} \equiv 0$ are also unstable. To see this consider the latter solution (the former being treated similarly). The linearized coefficient matrix is lower triangular with diagonal entries $\alpha-2 \beta \tilde{P}$ and $\alpha-\xi \tilde{P}$. From (A.5) it follows that

$$
\operatorname{av}[\alpha-\beta \tilde{P}]=0 .
$$

Thus the two Floquet exponents are

$$
\operatorname{av}[\alpha-2 \beta \tilde{P}]=-\operatorname{av}[\beta \tilde{P}]<0, \quad \operatorname{av}[\alpha-\xi \tilde{P}]>\operatorname{av}[\alpha-\beta \tilde{P}]=0 .
$$

The latter inequality implies instability.

It now follows from the results of de Mottoni and Schiaffino [7] that the remaining periodic solution $P_{1} \equiv P_{2} \equiv P>0$ is globally attracting.

The next and final theorem gives formulas for the Floquet exponents of a positive periodic solution of (A.1). 
Theorem 2. If $P_{1} \equiv P_{2} \equiv P>0$ is a periodic solution of $(A .1)$, then the Floquet exponents of the linearizea system associated with this solution are

$$
-p \operatorname{av}[\alpha], \quad-p \operatorname{av}[(\beta-\xi P)] .
$$

That at least one positive periodic solution exists is guaranteed by Corollary 1 . If $\xi(t) \leqslant \beta(t)$ for all $t$, but $\xi \neq \beta$, then it is unique and the two Floquet exponents in Theorem 2 are negative, as is consistent with the global attractivity guaranteed by Corollary 2 .

Proof. The linearization of (A.1) at $P_{1} \equiv P_{2} \equiv P$ is

$$
\begin{gathered}
x^{\prime}=e(t) x+d(t) y \\
y^{\prime}=d(t) x+e(t) y \\
e:=\alpha-(2 \beta+\xi) P, \quad d:=-\xi P .
\end{gathered}
$$

By adding these equations and then subtracting them, one finds that

$$
(x+y)^{\prime}=(e+d)(x+y), \quad(x-y)^{\prime}=(e-d)(x-y)
$$

and hence

$$
x \pm y=\left(x_{0} \pm y_{0}\right) \exp \left(\int_{0}^{t}(e \pm d) d s\right)
$$

where $x_{0}=x(0), y_{0}=y(0)$. The Floquet multipliers are the eigenvalues of the normalized fundamental solutions matrix $Y(t)$ evaluated at $t=p$. The fundamental solution matrix $Y(t)$ has columns generated by the solutions for which $x_{0}=1, y_{0}=0$ and $x_{0}=0, y_{0}=1$ respectively. The eigenvalues are the roots of the quadratic

$$
m^{2}-\operatorname{tr} Y(p) m+\operatorname{det} Y(p)
$$

A straightforward calculation using (A.7) shows that

$$
\begin{aligned}
\operatorname{tr} Y(p) & =\exp (p \operatorname{av}[e+d])+\exp (p \operatorname{av}[e-d]) \\
\operatorname{det} Y(p) & =\exp (2 p \text { av }[e]) .
\end{aligned}
$$

The eigenvalues or Floquet multipliers then turn out to be

$$
m=\exp (p \operatorname{av}[e+d]) \text { and } \exp (p \operatorname{av}[e-d]) .
$$

From (A.2) follows, since $P>0$,

$$
\operatorname{av}[\alpha]=\operatorname{av}[(\beta+\xi) P]
$$

Consequently from (A.6)

$$
\begin{aligned}
\operatorname{av}[e+d] & =\operatorname{av}[\alpha-2(\beta+\xi) P]=-\operatorname{av}[\alpha] \\
\operatorname{av}[e-d] & =\operatorname{av}[\alpha-2 \beta P]=\operatorname{av}[(\beta+\xi) P]-2 \operatorname{av}[\beta P] \\
& =-\operatorname{av}[(\beta-\xi) P]
\end{aligned}
$$

and the theorem is proved.

\section{References}

1. Butler, G. J., Hsu, S. B., Waltman, P.: A mathematical model of the chemostat with periodic washout rate. SIAM J. Appl. Math. 45, 435-449 (1985)

2. Christiansen, F. B., Fenchel, T. M.: Theories of populations in biological communities. Ecological Studies 20. Berlin, Heidelberg, New York: Springer, 1977 
3. Coddington, E. A., Levinson, N.: Theory of ordinary differential equations. New York: McGrawHill, 1955

4. Cushing, J. M.: Two species competition in a periodic environment. J. Math. Biol. 10, 385-400 (1980)

5. Cushing, J. M.: Periodic Kolmogorov systems. SIAM J. Math. Anal, 13, 811-827 (1982)

6. Cushing, J. M.: Periodic two-predator, one-prey interactions and the time sharing of a resource niche. SIAM J. Appl. Math. 44, 392-410 (1984)

7. de Mottoni, P., Schiaffino, A.: Competition systems with periodic coefficients: a geometric approach. J. Math. Biol. 11, 319-335 (1981)

8. Hale, J. K., Somolinos, A. S.: Competition for fluctuating nutrient. J. Math. Biol. 18, 225-280 (1983)

9 Harper, J. L.: The regulation of numbers and mass in plant populations. In: Lewontin, R. C. (ed.), Population biology and evolution. Syracuse University Press, 1968

10. Hutchinson, G. E.: The paradox of the plankton. Am. Naturalist 95, 137-143 (1961)

11. Koch, A. L.: Coexistence resulting from an alternation of density independent growth. J. Theor. Biol. 44, 372-386 (1974)

12. May, R. M.: Stability and complexity in model ecosystems. In: Monographs in Population Biology 6. Princeton, NJ: Princeton University Press, 1974

13. Namba, T.: Competitive co-existence in a seasonally fluctuating environment, $\mathbf{J}$. Theor. Biol. 111, 369-386 (1984)

14. Namba, T.: Bifurcation phenomena appearing in the Lotka-Volterra competition equations: a numerical study. Preprint

15. Pianka, E. R.: Ecology of the agamid lizard amphibolurus ilolepsis in Western Australia. Copeia 1971, 527-536 (1971)

16. Pianka, E. R.: Evolution ecology. New York: Harper and Row, 1978

17. Pielou, E. C.: Population and community ecology: principles and methods. New York: Gordon and Breach, 1978

18. Ricklefs, R. E.: Ecology. Newton, Mass.: Chiron Press, 1974

19. Rosenblat, S.: Population models in a periodically fluctuating environment. J. Math. Biol. 9, 23-36 (1980)

20. Sánchez, D. A.: Periodic environments, harvesting, and a Riccati equation. In: Lakshmikantham, V. (ed), Nonlinear phenomena in mathematical sciences. New York: Academic Press, 1982

21. Smith, H. L.: Competititive coexistence in an oscillating chemostat. SIAM J. Appl. Math. 40, 498-522 (1981)

22. Vesey-Fitzgerald, D. F.: Grazing succession among East African game animals. J. Mammalogy 41, 161-172 (1960)

23. Zaret, T. M., Rand, A. S.: Competition in tropical stream fishes: support for the competitive exclusion principle. Ecology 52, 336-342 (1971).

Received March 14/Revised June 18, 1986 